\title{
Nonreward anticipated: Effects on extinction runway performance in the rat*
}

\author{
M. J. HOMZIE \\ University of Virginia, Charlottesville, Virginia 22901
}

\begin{abstract}
Following a 47-day extinction procedure, the reinstatement of the cue previously associated with reward produced an immediate improvement in performance. The cue was the opportunity to traverse the alley following an "anticipated" nonrewarded runway trial. Moreover, the animals trained in this matter exhibited daily increments in performance during the initial phase of extinction testing. The results were interpreted as consistent with the notion that the difference in extinction performance of one group as compared to another does not necessarily reflect the relative "strengths" of the instrumentally acquired habits. Instead, it probably indicates the degree of similarity of the extinction testing procedure to the acquisition training condition previously associated with reinforcement for the two individual groups.
\end{abstract}

Following an extensive experimental extinction procedure, rats typically refuse to run and even resist being placed into the runway. Within the context of the sequential aftereffects hypothesis (e.g., Capaldi, 1966, 1967), extinction is viewed as a transfer test situation. During acquisition, the initial phase of an experiment, the animal learns to run in the presence of specific stimuli: extinction, the transfer task, consists of testing for this learned behavior under conditions differing from those that prevailed during acquisition training. Certainly, the dominant class of stimuli changed (from acquisition to extinction) are those related to the reinforcement conditions. The extinction procedure, however, can be conducted such as to produce a large decrement in a rat's running speed but not remove the contingency established during acquisition between particular stimuli and subsequent reinforcement events (e.g., Homzie, Gohmann, \& Hall, 1971).

In the present experiment during acquisition training, one group of animals was provided with consistent cues as to forthcoming reinforcement and nonreinforcement events: the reinforcement schedule was such that, following a nonreinforced runway trial, all subsequent opportunities to locomote in the apparatus were rewarded. The extinction procedure consisted of administering only one nonreinforced runway trial per day, thereby not breaking the contingency established during acquisition training between "the animal's opportunity to locomote in the alleyway following nonreinforced trials" and subsequent reinforced runway trials. According to the aftereffects hypothesis (Capaldi, 1966, 1967), it would be expected that the reinstatement of the cue previously associated with

*This research was supported in part by National Science Foundation Grant GB-13341. The paper was written during the author's research leave: the generous support of the Laboratory of Experimental Psychology, University of Sussex, is gratefully acknowledged. Appreciation is expressed to T. Gohmann and S. W. Hall, Jr. for their assistance in data collection and analysis. reward (i.e., the opportunity to traverse the alleyway following a nonreinforced trial) would result in an immediate improvement in runway performance (cf. Homzie et al, 1971; Homzie \& Rudy, 1971). A control group was included that received the same daily number of reinforcement and nonreinforcement runway trials, but the acquisition training sequence was such that this group was not provided with reliable cues as to forthcoming goal events.

\section{METHOD}

\section{Subjects}

The Ss were 17 experimentally naive Sprague-Dawley and Long-Evans hooded male rats 90-110 days old and weighing $200-225 \mathrm{~g}$ at the start of pretraining. The animals were randomly assigned to the two groups and were maintained on a 23-h food-deprivation schedule.

\section{Apparatus}

The apparatus consisted of a straight alley $113 \times 10.1 \times 10.1 \mathrm{~cm}$ deep. The sides of the alley were constructed of plywood and lined with $3.2-\mathrm{mm}$ Plexiglas. For all sections of the runway, a gray cardboard filler was placed between the Plexiglas lining and the plywood. The floor of the alley was constructed of $6.4-\mathrm{mm}$ aluminum bars spaced $1 \mathrm{~cm}$ apart. The startbox was $30.5 \mathrm{~cm}$ long and $5 \mathrm{~cm}$ wide, separated from the runway section by a Plexiglas guillotine door. The goalbox section was $22.9 \mathrm{~cm}$ long and separated from the rest of the runway by a Plexiglas guillotine door that was used to prevent retracing. The entire apparatus was covered by Plexiglas hinged to the runway.

Starting time was measured photoelectrically from the opening of the startbox door to a point $15.2 \mathrm{~cm}$ within the runway; running and goal time were measured over the next two $30.4-\mathrm{cm}$ segments of the apparatus, respectively.

\section{Procedure and Experimental Design}

The first day after their arrival, the animals were caged individually and allowed free access to food and water. On Day 8 the $S s$ were handled in pairs for $5 \mathrm{~min}$ and the 23 -h food-deprivation schedule was initiated. On Days 9-11 the rats 


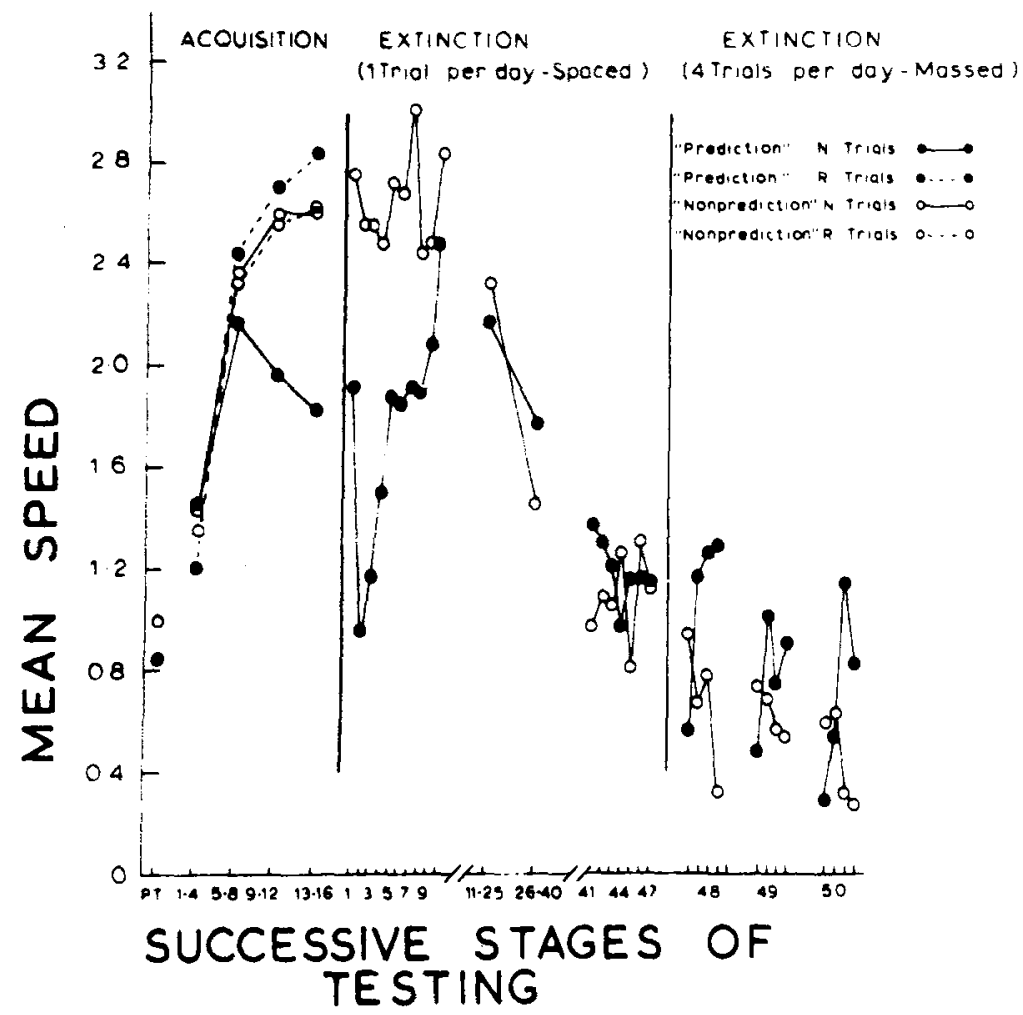

Fig. 1. Mean running speed performance as a function of successive stages of training.

were handled for $10 \mathrm{~min}$ in squads of six on a metal tray where 97-mg Noyes pellets were available. On Days 12 and 13, pairs of rats were given exposure to the runway for $2 \frac{1}{2} \mathrm{~min}$, the recording apparatus was connected, and the start- and goalbox doors were manipulated while the animals were in the runway. The following day the Ss were given two direct placements into the goalbox, baited with four 97-mg Noyes pellets. On the last day of pretraining, the rats were given two runway acquisition trials, receiving as a reward four $97-\mathrm{mg}$ pellets.

During the 16-day acquisition training period, each of the eight animals in the "prediction" group received an initially nonrewarded runway (N) trial, followed equally often by zero, one, two, of three reinforced runway (R) trials. Thus, for this group the first daily placement into the apparatus "signaled" nonreward, whereas all subsequent opportunities to traverse the runway were reinforced, i.e., the compound stimulus, consisting of a prior goal event and a $15-\mathrm{sec}$ intertrial interval, predicted $\mathrm{R}$ trials, whereas a 24 -h intertrial interval indicated a forthcoming $\mathrm{N}$ trial. In this manner, the rats could anticipate the outcome of all acquisition training runway trials. The nine animals in the other group received daily the same number of $\mathrm{N}$ and $\mathrm{R}$ trials as the previously mentioned rats. For these animals, however, the last trial of the day was nonreinforced. Since each training session could involve one, two, three, or four trials, these Ss could not anticipate the daily sequence of reinforcement events. All animals on $\mathrm{R}$ trials received as a reward four $97-\mathrm{mg}$ Noyes pellets. The rats were confined for $15 \mathrm{sec}$ in the empty goalbox on the $N$ trials. During extinction testing, all animals were administered a single $N$ trial each day for 47 days; the following 3 days the rats received four $\mathbf{N}$ trials daily. Throughout the entire experiment, on days that the animals received more than one runway trial, a $15-\mathrm{sec}$ intertrial interval was maintained.

\section{RESULTS AND DISCUSSION}

The response latencies were converted to speed scores (one/latency) and these transformed scores were used in all subsequent analyses. Only the data from the run segment of the apparatus are presented, because this measure was representative of the results obtained with all three measures.

The mean running speed performance, of the two groups is presented in Fig. 1 as a function of successive stages of testing. On the two $\mathrm{R}$ trials administered on the final day of pretraining (point PT on the abscissa), the groups performed similarly, indicating that there was little initial difference in the running speed of the two groups of rats. As expected, during acquisition training the rats that could anticipate the nature of forthcoming goal events ran statistically faster on $\mathbf{R}$ trials than on $\mathbf{N}$ trials (Wilcoxon test for correlated scores, $p<.01$ ), whereas the "nonprediction" group performed similarly on both types of trials. Collapsing the $16 \mathrm{~N}$ trials and the $24 \mathrm{R}$ trials presented each of the groups during acquisition showed that there was no overall difference in mean running speed performance.

As depicted in Fig. 1, over the first 10 days of extinction testing the prediction group performed reliably poorer than the nonprediction group (Mann-Whitney $\mathrm{U}=8, \mathrm{p}<.02$ ). This result is not too surprising; in acquisition training the first trial always had been an $\mathrm{N}$ trial, and the prediction group was merely performing in extinction in accordance with the reinforcement contingency established during acquisition training. After the 10th day, the differences in the single-trial extinction running speed performances of the two groups were quite small and not statistically reliable.

The unexpected extinction result was the daily 
improvement in the prediction group's runway performance. The mean running speed of every animal in this group was faster, for example, on Days 6-10 than on the first 5 days of extinction testing $(T=0, p<.01)$. It should be noted that this group was the one in which every first trial was nonrewarded during initial training. A possible post hoc analysis is that the single-trial extinction procedure did not immediately break the contingency acquired in acquisition-that $\mathrm{R}$ trials would follow a single $\mathrm{N}$ trial. Thus, the rise in the extinction curve could reflect an increasing expectation that $R$ trials would follow the single $\mathrm{N}$ trial, whereas the latter decline in running speed would reflect the eventual weakening of this acquisition-acquired contingency. Recall that the nonprediction animals also had acquisition training with single- $\mathrm{N}$-trial days but that $\mathrm{R}$ trials initiated three-fourths of this group's daily training sequences. Consequently, the successive single- $\mathrm{N}$-trial days presented during extinction could be expected to be more disruptive to this group's expectation that reinforcement days were eventually forthcoming.

Recently, similar results have been obtained with an experimental condition in which only an $\mathrm{N}$ trial administered in a particular manner (no confinement in the empty goalbox) could initiate the daily acquisition training sequence (Kendall \& Homzie, 1973). Extinction testing with repeated presentations of this type of $\mathrm{N}$ trial resulted in an initial decrement in runway performance, followed by subsequent improvements in running speed. Thus, possibly this highly unusual finding of spontaneous and consistent improvement in extinction runway performance is dependent in some manner on the animal's prior training experience that $\mathbf{R}$ trials eventually follow a particular type of $\mathrm{N}$ trial.

The last segment of Fig. 1 presents the trial by trial extinction performance for the days that the animals were switched to receiving four $\mathrm{N}$ trials. An analysis of each animal's trial by trial massed extinction running speed performance resulted in a significant Groups by Trials interaction $(F=1.95, \mathrm{df}=11 / 165$, $p<.05$ ). As indicated in the figure, generally the prediction group ran slowly on the first daily trial but exhibited a large increment in running speed on the second, third, and fourth daily trials $(\mathrm{T}=1, \mathrm{p}<.05$, Day 48 only). In contrast, the nonprediction group displayed the more typical within-day decline in runway performance. These results are consistent with the finding that animals administered the acquisition training schedule NR each day are more resistant to massed extinction than animals trained with the sequence RN (Mackintosh \& Little, 1970). In the present experiment, however, this effect "persisted" through a spaced extinction procedure and was observed only on the trials following the first daily $\mathrm{N}$ trial. Recall that, for the prediction group, an initial $\mathrm{N}$ trial followed by subsequent trials always resulted in reinforcement.
The first 47 days of extinction merely involved single $\mathrm{N}$ trials; consequently, the contingency previously established between an initial $\mathrm{N}$ trial and subsequent opportunities to locomote in the runway was not broken by the extensive testing procedure. Thus, by taking advantage of the acquisition reinforcement contingencies, it was possible to administer to rats 47 days of "spaced" extinction (at the end of which many animals refused to locomote in the runway), switch to "massed" extinction, and observe an enhancement of running speed on the appropriate daily trials.

These results are in agreement with other work indicating that the reinstatement of a cue previously associated with reward can result in an immediate improvement in extinction performance (Homzie et al, 1971; Homzie \& Rudy, 1971), but in this case the cue was somewhat unusual in that it was the animal's opportunity to locomote in the runway following an initially nonreinforced trial. This research indicates that animals are responding in extinction in accordance with the reinforcement contingencies established during acquisition training. This finding should not be particularly surprising. What has not been fully appreciated, however, is the extent to which extinction performance indicates exactly what was learned during the initial training. Capaldi, in his recent elaboration of the aftereffects hypothesis (e.g., Capaldi, 1966, 1967), points out that the extinction performance exhibited by two groups trained under different acquisition conditions does not indicate the relative "strengths" of the learned habits. Instead, it probably represents the degree of similarity of the extinction testing procedure to the acquisition training conditions previously associated with reinforcement for the two individual groups.

\section{REFERENCES}

Capaldi, E. J. Partial reinforcement: A hypothesis of sequential effects. Psychological Review, 1966, 73, 459-477.

Capaldi, E. J. A sequential hypothesis of reward variables: Partial reward and magnitude of reward. In K. W. Spence and J. T. Spence (Eds.), Psychology of learning and motivation. Vol. 1. New York: Academic Press, 1967.

Homzie, M. J., Gohmann, T., \& Hall, S. W., Jr. Runway performance in rats as determined by the predictive value of intertrial reinforcements. Journal of Comparative \& Physiological Psychology, 1971, 74, 90-95.

Homzie, M. J., \& Rudy, J. W. Effect on runway performance of reinforcement contingencies established to empty goal box placements. Learning \& Motivation, 1971, 2, 95-101.

Kendall, P. C., \& Homzie, M. J. Acquisition and transfer effects of cued and non-cued double alternation schedules with rats. Learning \& Motivation, $1973,4,459-470$.

Mackintosh, N. J., \& Little, L. Effects of difterent patterns of reinforcement on performance under massed or spaced extinction. Psychonomic Science, 1970, 20, 1-2.

(Received for publication June 18, 1973; revision accepted October 16, 1973.) 